\title{
Non-rigid Image Registration with Uniform Spherical Structure Patterns
}

\author{
Shu Liao and Albert C.S. Chung \\ Lo Kwee-Seong Medical Image Analysis Laboratory, \\ Department of Computer Science and Engineering, \\ The Hong Kong University of Science and Technology, Hong Kong \\ liaoshu@cse.ust.hk, achung@cse.ust.hk
}

\begin{abstract}
Non-rigid image registration is a challenging task in medical image analysis. In recent years, there are two essential issues. First, intensity similarity is not necessarily equivalent to anatomical similarity when the anatomical correspondences between subject and template images are established. Second, the registration algorithm should be robust against monotonic gray-level transformation when aligning anatomical structures in the presence of bias fields. In this paper, a new feature based non-rigid registration method is proposed to deal with these two problems. The proposed method is based on a new type of image feature, called Uniform Spherical Structure Pattern (USSP). USSP encodes voxel-wise interaction information and geometric properties of anatomical structures. It is computationally efficient, rotation invariant and theoretically monotonic gray-level transformation invariant. The USSP feature is integrated with the Markov random field (MRF) discrete labeling framework to define energy function for registration in this paper. If the segmentation results are available, explicit anatomical correspondence can be established as an additional energy term. The energy function is optimized via the alpha-expansion algorithms. The proposed method is compared with three widely used non-rigid registration methods on both simulated and real databases obtained from BrainWeb and IBSR. Experimental results demonstrate that the proposed method achieves the highest registration accuracy among all the compared methods.
\end{abstract}

\section{Introduction}

Non-rigid image registration plays an important role in medical image analysis. Its clinical applications include, but not limited to, anatomical analysis and statistical parametric mapping. Many novel methods have been proposed during the last decade. They can be broadly classified into three categories: landmark based, intensity based and feature based registration methods. Landmark based registration methods extract anatomical features from manually located landmark points. Transformations are estimated based on such anatomical features [1]2]. Landmark based registration methods use prior knowledge obtained from manually placed landmark points and thus they are usually computationally efficient. 
However, to produce accurate registration results, these methods need sufficient number of landmark points and therefore require additional burdens. Intensity based registration methods define a similarity measure metric evaluated from voxel intensity distributions to guide the registration process [34. An essential issue related to intensity based registration methods is intensity similarity is not necessarily equivalent to anatomical similarity. Feature based registration methods use feature vectors as signatures for each voxel. Then the registration process is formulated as a feature matching and optimization problem.

Despite the fact that many aforementioned methods have been proposed to tackle the non-rigid registration problem, two challenges arise in recent years. First, the goal of non-rigid image registration is to establish anatomical correspondence between the template and the subject images. Using absolute voxel intensity values alone to characterize anatomical properties may be insufficient, as pointed out in [5], and can make the similarity measure function stuck at local minima. As such, effective anatomical region descriptor is needed. Second, the registration algorithm should be robust against monotonic gray-level bias fields, which commonly exist during the imaging process. Otherwise, the registration algorithm may prefer to align the bias fields instead of aligning anatomical structures, as discussed in [6].

Therefore, we are motivated to propose a new feature based non-rigid registration method which can accurately capture the geometric properties of the anatomical structures and theoretically robust against the monotonic gray-level bias fields. There are three main contributions in this paper. (i) A new anatomical region descriptor, called uniform spherical structure pattern (USSP) is designed to capture the anatomical geometry of the input images. (ii) The USSP descriptor is theoretically invariant to monotonic gray-level bias fields and image rotation. (iii) If the segmentation results of input images are available, an explicit anatomical energy term based on the Fisher's separation criteria (FSC) is proposed to measure the distance of different tissue classes between the subject and the template images. Markov random field (MRF) discrete labeling has been shown to be a robust framework to model the non-rigid registration process in recent years 78 . In this paper, the USSP feature is integrated with the MRF labeling framework to define the energy function which guides the registration process. The $\alpha$-expansion algorithm is used to minimize the MRF energy function. The proposed method is evaluated on both the simulated and real 3D databases obtained from BrainWeb and IBSR and compared with three widely used registration algorithms. It is observed that the proposed method achieves the highest registration accuracy among all the compared methods.

\section{Feature Extraction with Uniform Spherical Structure Patterns}

In this section, we describe the design details of the uniform spherical structure pattern (USSP) region descriptor, analyze its properties, and show how to use USSP to extract anatomical features from input images. 


\subsection{Basic Spherical Structure Patterns}

Suppose for a given input image $G$, for each voxel $v \in G$, we define a sphere $S_{v}$, which is centered at $v$ with radius $R . N_{v}$ samples are uniformly taken on the surface of $S_{v}$ using the sampling method proposed in 9]. Samples which do not fall exactly into the image grid are interpolated by using the trilinear interpolation method. Then, for each voxel $i$ on the surface of $S_{v}$, it is thresholded to binary numbers "0" or "1" by using the Equation 1 .

$$
B_{i}=\left\{\begin{array}{l}
0, \text { if } I_{i}<I_{v}, \\
1, \text { if } I_{i} \geq I_{v},
\end{array}\right.
$$

where $I_{i}$ is the intensity of voxel $i$, and $I_{v}$ is the intensity of the center voxel $v$.

The thresholded surface represents the geometric features surrounding the voxel $v$ as the binary values reflect the voxel-wise interactions between the neighboring voxels and the center voxel. The thresholded surface is called the basic spherical structure pattern (BSSP). Local binary pattern [10] is a special case of BSSP in the 2D case. The formal definition of BSSP is given as follows:

Definition 1: Basic spherical structure pattern (BSSP) is the thresholded spherical surface obtained from the original spherical neighborhood centered at the reference voxel by using the Equation 1 .

BSSP is monotonic gray-level transformation invariant because the neighboring voxels are converted to the binary digits by comparing their intensity values with the center voxel intensity. As long as the relative difference between two voxel intensity values does not change, the thresholded surface remains the same.

\subsection{Uniform Spherical Structure Patterns}

Though the BSSP proposed in Section 2.1 is monotonic gray-level transformation invariant, there are many types of BSSP which are too sparse to reliably reflect the anatomical features of input images. In this section, we define the uniform spherical structure pattern (USSP) which is a subset of BSSP and represents fundamental image structures. USSP is defined as follows:

Definition 2: Uniform spherical structure patterns (USSP) are basic spherical structure patterns (BSSP), which have AT MOST two continuous regions of " $0 " s$ and "1"s.

For example, Figures 1 (a) and (b) are USSPs, while Figure 1 (c) is not a USSP. The pseudo code of determining whether a BSSP is a USSP or not is presented in the Algorithm 1. The time complexity of the Algorithm 1 is analyzed as follows. Finding the largest connected component by using the BFS algorithm needs $O(N)$ time. Operation 3 also needs $O(N)$ time in the worst case. Other operations take constant computation time. Therefore, the Algorithm 1 takes $O(N)$ time, which is a linear time algorithm. 


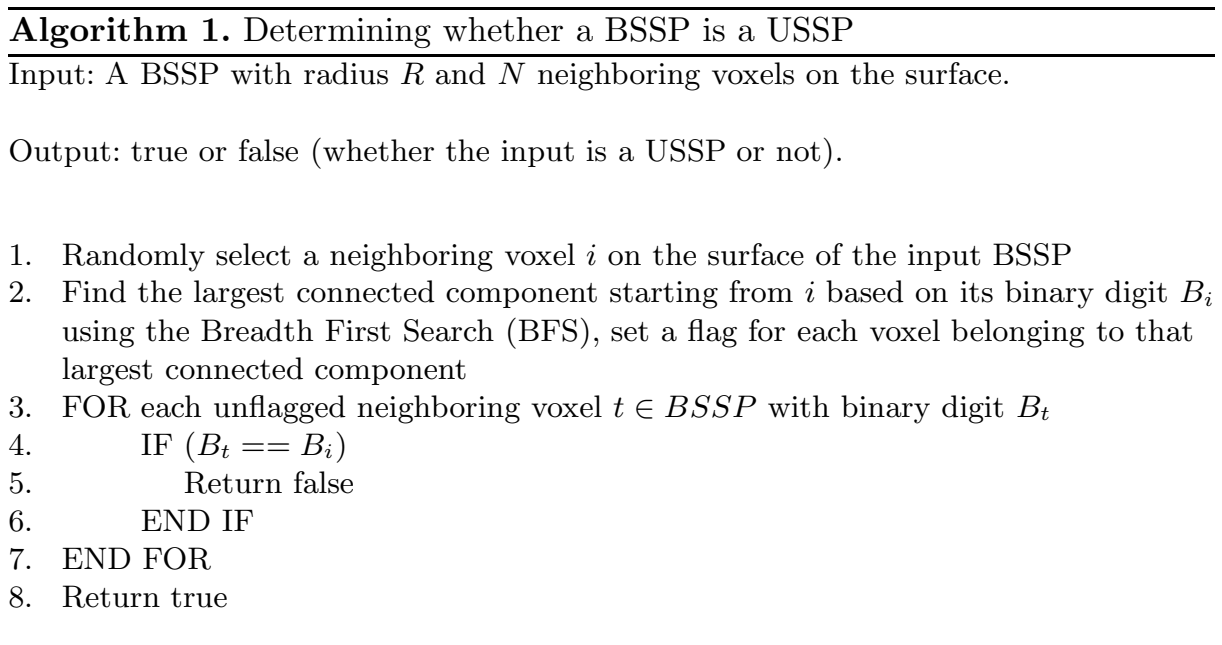

USSP contains important physical meanings regarding the fundamental image structures. For example, Figure 1 (a) represents a dark spot as all the surrounding voxels' intensity values are higher than or equal to the intensity of the center voxel. Figure 1 (b) reflects that there is an edge along the center voxel as half of the neighboring voxels' intensity values are lower than the intensity of the center voxel and lie on the same continuous region, while the other half of the neighboring voxels just act as the opposite. All the non-uniform spherical structure patterns are considered as a single type of image structure in this paper.

To further illustrate that USSP encodes dominant information and represents the fundamental image structures, Figures 2 (a) to (c) plot the proportions of USSPs among all the BSSPs with different parameters for 20 image volumes

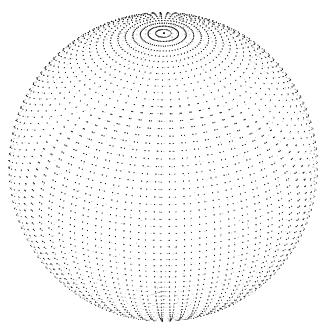

(a)

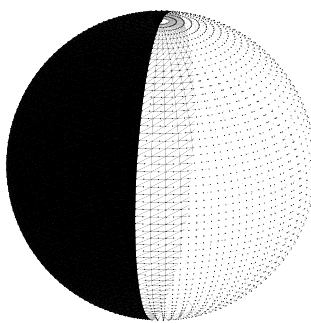

(b)

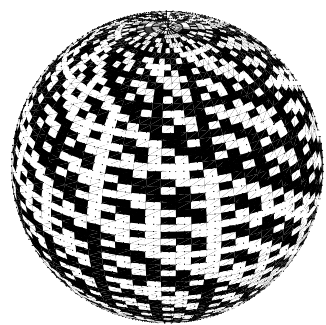

(c)

Fig. 1. (a) An example of USSP, with all "1"s (white region) on the surface. There is only one continuous region of all "1"s on the surface; (b) An example of USSP, with half the surface of "0"s (black region) and half the surface of "1"s (white region). There are two continuous regions (i.e., one with "0"s, the other with "1"s); (c) An example of non-uniform SSP. There are more than two continuous regions with "0"s and "1"s on the surface. 


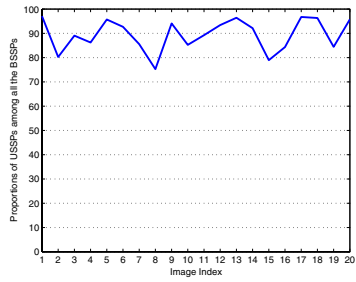

(a)

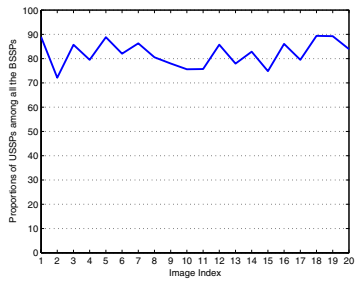

(b)

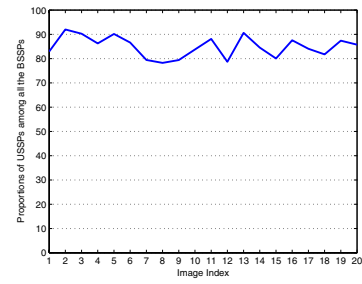

(c)

Fig. 2. Proportions (in \%) of USSPs among all the BSSPs of 20 image volumes obtained from IBSR, with parameters: (a) Radius = 1, Number of Neighboring Samples = 25; (b) Radius $=2$, Number of Neighboring Samples $=36$; (c) Radius $=3$, Number of Neighboring Samples $=49$

obtained from IBSR 1 . It is observed from Figure 2 that USSPs have dominant proportions among all the BSSPs (i.e., mostly over $80 \%$ ) with different parameters. Therefore, USSP can reliably capture the anatomical properties of the input image volumes. In the rest of the paper, we will only focus on extracting USSP features from the input images.

\subsection{Feature Extraction of Rotation Invariant USSPs}

Though USSP contains dominant information and represents fundamental images structures, it is still not rotation invariant up to the current stage. When the image rotates, the positions of the binary digits on the surface of USSP will be shifted accordingly. As pointed out in [11, rotation invariance is a desired property for feature based non-rigid registration methods.

Therefore, we design an algorithm to extract rotation invariant USSP features. It is observed that no matter how the image rotates, the region areas of " 0 " $s$ and "1"s (i.e., the number of voxels belonging to "0"s and "1"s) of a USSP pattern do not change. Therefore, the number of voxels belonging to "0" or " 1 " binary digits can be used to denote the types of USSP, which should be invariant to rotation. In this paper, the type ID of the USSP is determined by the number of "0"s in the USSP. All the non-uniform BSSPs are treated as a single type pattern. Algorithm 2 presents the procedure for calculating rotation invariant USSP signatures for each voxel $v$ from a local cubic square window $W$ centered at $v$, assuming that the radius $R$ and number of neighboring samples $N$ are given. The time complexity of the Algorithm 2 is $O(|G| \times|W| \times N)$, where $|G|$ is the number of voxels of the input image $G,|W|$ is the window size and $N$ is the number of neighboring samples used for USSP.

It should be noted that Algorithm 2 only takes care of the area of "0" region to determine the USSP type. A more detailed USSP type classification can be

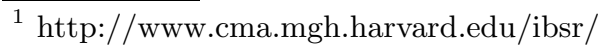




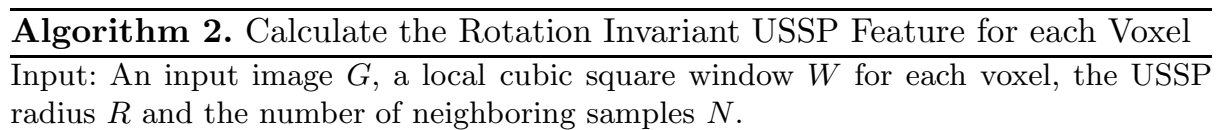

Output: A vector image $K$, each voxel is represented by a USSP signature.

1. FOR each voxel $v \in G$

2. $\quad$ SubVolume $=(W$ center at $v)$

3. Initialize a new feature histogram, $H[0 \ldots(N+1)]=0$

4. FOR each voxel $t \in$ SubVolume

5. Calculate its corresponding BSSP $Q_{t}$ with parameters $R$ and $N$

6. Determine whether $Q_{t}$ is a USSP or not using Algorithm 1

7. IF $Q_{t}$ is a USSP

8. $\quad$ PatternID $=$ Number of " 0 "s in $Q_{t}$

9. $\quad H[$ PatternID $]=H[$ PatternID $]+1$

$10 . \quad$ ELSE

11. $H[N+1]=H[N+1]+1$

12. $\quad$ END IF

13. END FOR

14. Normalize $H[0 \ldots(N+1)]$ such that $\sum_{i=0}^{N+1} H[i]=1$

15. $K(v)=H[0 \ldots(N+1)]$

16. END FOR

17. Return $K$

achieved if we also take care of the shape of the "0" region. However, there are too many possible shape combinations and will result in unstable anatomical feature description as some of the USSP occurrences are too small. This makes the USSP feature histogram too sparse to reliably mirror the anatomical properties of the input images. Radius $R$ in the Algorithm 2 affects the scale of interest to extract the anatomical structures. In this paper, the radius $R$ is determined by adopting the best scale selection principle proposed in [12].

The rotation invariant USSP feature also preserves the monotonic gray-level transformation invariant property of BSSP. Figure 3 shows an example. Figure 3 (a) is an image slice obtained from BrainWeb with no bias field distortion. Figure 3 (b) is a type-A bias field obtained from BrainWeb with $40 \%$ inhomogeneity. Figure 3 (c) is the resulting bias field distorted image. The region inside the green rectangle is the region of interest (ROI). The rotation invariant USSP feature vectors with 49 neighboring samples are extracted from the ROI by treating the ROI as the SubVolume in the Algorithm 2 Figures 3 (f) and (g) are the rotation invariant USSP feature vectors extracted from the ROI before and after bias field distortion respectively. For comparison purpose, the intensity histograms of the ROI before and after bias field distortion are also shown in Figures 3 (d) and (e). It is observed that the intensity histograms of ROI before and after bias field distortion have large variations. The rotation invariant 


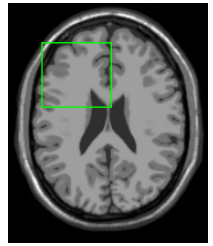

(a)

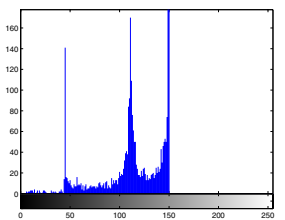

(d)

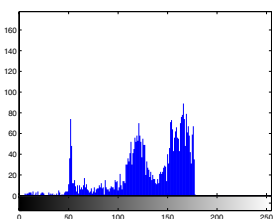

(e)

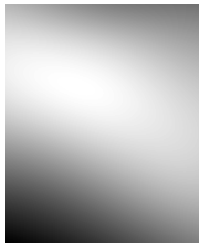

(b)

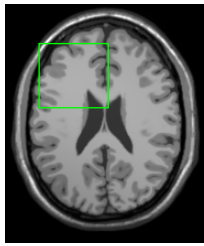

(c)

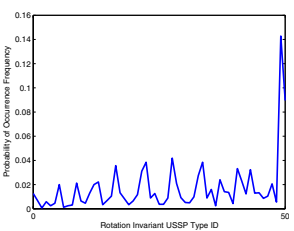

(f)

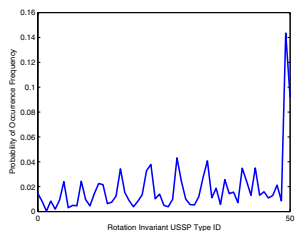

$(\mathrm{g})$

Fig. 3. (a) The original image; (b) The bias field of $40 \%$ inhomogeneity; (c) Resulting image after applying the bias field in (b) to the original image in (a); (d) Intensity histogram of ROI (green rectangle region in (a)) in the original image; (e) Intensity histogram of ROI (green rectangle region in (c)) in the bias field distorted image; (f) Rotation invariant USSP feature of ROI in the original image; (g) Rotation invariant USSP feature of ROI in the bias field distorted image

USSP feature vectors extracted from ROI before and after bias field distortion are almost the same (i.e., just with slight variations). Therefore, the robustness of the USSP features against bias field distortion is implied.

\section{MRF Labeling Formulation for Registration}

Recently, the MRF discrete labeling framework is shown to be able to robustly model the non-rigid registration process [7/8. In this paper, the USSP feature is integrated with the MRF discrete labeling framework to define the energy function. An explicit anatomical correspondence energy term is also proposed based on the Fisher's separation criteria (FSC) [1314 if the segmentation results of the input images are available.

The general form of the MRF energy function by considering clique size order up to two is expressed as:

$$
\begin{aligned}
E_{f} & =E_{\text {data }}+E_{\text {smoothness }} \\
& =\sum_{p \in \Omega} D_{p}\left(l_{p}\right)+\sum_{(p, q) \in N} V_{p, q}\left(l_{p}, l_{q}\right),
\end{aligned}
$$

where $\Omega$ is the set of voxels, $N$ is the neighborhood system defined in $\Omega$. In this paper, the 4-connected neighborhood system is used. $D_{p}\left(l_{p}\right)$ is the energy function, which penalizes the cost of assigning label $l_{p}$ to voxel $p$, and $V_{p, q}\left(l_{p}, l_{q}\right)$ penalizes the cost of label discrepancy between two neighboring voxels. 
The deformation space is quantized to transform the registration problem to the MRF labeling problem. A discrete set of labels $L \in\left\{l^{1}, l^{2}, \ldots, l^{n}\right\}$ is defined. Each label $l^{i}(1 \leq i \leq n)$ corresponds to a displacement vector $\boldsymbol{d}_{\boldsymbol{i}}$. Each label assignment $l_{p}$ to voxel $p$ denotes moving $p$ to a new position according to the corresponding displacement vector $\boldsymbol{d}_{\boldsymbol{l}_{p}}$. The quantization step in [7] is adopted in this paper. Each voxel can be displaced off the original position bounded by a discretized window $\Psi=\{0, \pm s, \pm 2 s, \ldots, \pm w s\}^{d}$ of dimension $d$.

The energy function $D_{p}\left(l_{p}\right)$ is defined based on the USSP features as:

$$
\begin{aligned}
D_{p}\left(l_{p}\right) & =D_{p}\left(G_{\text {template }}(\boldsymbol{p}), G_{\text {subject }}\left(\boldsymbol{p}+\boldsymbol{d}_{\boldsymbol{l}_{\boldsymbol{p}}}\right)\right. \\
& =D_{p}\left(K_{\text {template }}(\boldsymbol{p}), K_{\text {subject }}\left(\boldsymbol{p}+\boldsymbol{d}_{\boldsymbol{l}_{\boldsymbol{p}}}\right)\right) \\
& =J S D\left(K_{\text {template }}(\boldsymbol{p}) \| K_{\text {subject }}\left(\boldsymbol{p}+\boldsymbol{d}_{\boldsymbol{l}_{\boldsymbol{p}}}\right)\right),
\end{aligned}
$$

where $G_{\text {template }}$ is the template image, $G_{\text {subject }}$ is the subject image, $K_{\text {template }}$ and $K_{\text {subject }}$ are the USSP feature vector images of $G_{\text {template }}$ and $G_{\text {subject }}$ respectively obtained via the Algorithm [2, $J S D(\cdot)$ denotes the Jensen-Shannon divergence. Therefore, based on the Equation 3, the data term $E_{\text {data }}$ at iteration $t$ is defined as:

$$
E_{\text {data }}^{t}=\sum_{p \in \Omega} J S D\left(K_{\text {template }}(\boldsymbol{p}) \| K_{\text {subject }}^{t-1}\left(\boldsymbol{p}+\boldsymbol{d}_{\boldsymbol{l}_{\boldsymbol{p}}}\right)\right),
$$

where $K_{\text {subject }}^{t-1}$ denotes the USSP feature vector image of the subject image resulting from the previous transformation prior to iteration $t$.

The piece-wise truncated absolute distance is adopted as the smoothness potential function:

$$
V_{p, q}\left(l_{p}, l_{q}\right)=\min \left(\lambda,\left|\boldsymbol{d}_{\boldsymbol{l}_{p}}-\boldsymbol{d}_{\boldsymbol{l}_{\boldsymbol{q}}}\right|\right),
$$

where $\lambda$ is a constant represents the maximum penalty. The truncated absolute distance is a metric as stated in [15].

If the segmentations of the template image and the subject image are available, an explicit anatomical energy term can be established as an additional energy term based on FSC. Suppose that the input images are segmented into $c$ classes of tissues. Let $V_{i}^{T}(1 \leq i \leq c)$ and $V_{i}^{S}(1 \leq i \leq c)$ denote the volumes of voxels belonging to tissue class $i$ of the template and the subject images, $N_{i}^{T}$ and $N_{i}^{S}$ denote the numbers of voxels in $V_{i}^{T}$ and $V_{i}^{S} \cdot \boldsymbol{F}_{\boldsymbol{i}, \boldsymbol{j}}^{\boldsymbol{T}}$ and $\boldsymbol{F}_{\boldsymbol{i}, \boldsymbol{j}}^{\boldsymbol{S}}$ denote the USSP feature vectors from the $j$ th voxel of the $i$ th class tissue of the template and the subject images at current iteration. We first calculate the mean of the $i$ th class tissue of the template and the subject images as: $\boldsymbol{m}_{\boldsymbol{i}}^{\boldsymbol{T}}=\frac{1}{N_{i}^{T}} \sum_{k=1}^{N_{i}^{T}} \boldsymbol{F}_{\boldsymbol{i}, \boldsymbol{k}}^{\boldsymbol{T}}$, $\boldsymbol{m}_{\boldsymbol{i}}^{\boldsymbol{S}}=\frac{1}{N_{i}^{S}} \sum_{k=1}^{N_{i}^{S}} \boldsymbol{F}_{\boldsymbol{i}, \boldsymbol{k}}^{\boldsymbol{S}}$.

Then, according to the principles of FSC [13, the 1-D space of the projected feature vector which can maximize the separability of the feature vector cluster of $V_{i}^{T}$ and $V_{i}^{S}$ is given by:

$$
y_{z}=\left(\boldsymbol{m}_{\boldsymbol{i}}^{\boldsymbol{T}}-\boldsymbol{m}_{\boldsymbol{i}}^{\boldsymbol{S}}\right)^{T} \mathbf{S}^{-1} \boldsymbol{F}_{\boldsymbol{z}}
$$


where $\mathbf{S}^{-1}$ is the inverse of the pooled covariance matrix, $\boldsymbol{F}_{\boldsymbol{z}}$ is the USSP feature vector of voxel $z$ belonging to the $i$ th class tissue of either the template image or the subject image. Therefore, the 1-D projections of $\boldsymbol{m}_{\boldsymbol{i}}^{\boldsymbol{T}}$ and $\boldsymbol{m}_{\boldsymbol{i}}^{\boldsymbol{S}}$ according to the Equation 6 are:

$$
\begin{aligned}
K_{i}^{T} & =\left(\boldsymbol{m}_{\boldsymbol{i}}^{\boldsymbol{T}}-\boldsymbol{m}_{\boldsymbol{i}}^{\boldsymbol{S}}\right)^{T} \mathbf{S}^{-1} \boldsymbol{m}_{\boldsymbol{i}}^{\boldsymbol{T}} \\
K_{i}^{S} & =\left(\boldsymbol{m}_{\boldsymbol{i}}^{\boldsymbol{T}}-\boldsymbol{m}_{\boldsymbol{i}}^{\boldsymbol{S}}\right)^{T} \mathbf{S}^{-1} \boldsymbol{m}_{\boldsymbol{i}}^{\boldsymbol{S}}
\end{aligned}
$$

This 1-D projection maximizes the following FSC measure function [13] of tissue class $i$ of the template image and the subject image:

$$
f_{i}=\frac{\left|K_{i}^{T}-K_{i}^{S}\right|}{\sqrt{\left(\sigma_{i}^{T}\right)^{2}+\left(\sigma_{i}^{S}\right)^{2}}}
$$

where $\sigma_{i}^{T}$ and $\sigma_{i}^{S}$ are the standard deviations of the projected USSP feature vector belonging to the $i$ th tissue class of the template image and the subject image.

When the FSC measure function in Equation 9 is minimized, the anatomical similarity of tissue class $i$ is maximized. The explicit anatomical energy term is defined by summing up the FSC measure functions defined in the Equation 9 of all the tissue classes. Therefore, now the total energy function becomes:

$$
\begin{aligned}
E_{f} & =E_{\text {data }}+E_{\text {smoothness }}+E_{\text {anatomy }} \\
& =\sum_{p \in \Omega} D_{p}\left(l_{p}\right)+\sum_{(p, q) \in N} V_{p, q}\left(l_{p}, l_{q}\right)+\sum_{i=1}^{c} f_{i} .
\end{aligned}
$$

In order to minimize the energy function defined in Equations 2 and 10, the alpha-expansion algorithm [15] is adopted.

\section{Experimental Results}

The proposed method is evaluated by performing non-rigid registration experiments on both the simulated and real 3D datasets obtained from BrainWeb2 and $\mathrm{IBSR}^{3}$ respectively. It is also compared with three state-of-the-art algorithms: FFD 3, Demons 44 and HAMMER [1]. In all the experiments, the local cubic square window $W$ in the Algorithm 2 was set to $16 \times 16 \times 16$, and number of neighboring samples $N$ of each USSP was 49 . The $3 \mathrm{D}$ displacement window for the proposed method was $\Psi=\{0, \pm 1, \pm 2, \ldots, \pm 12\}^{3}$. The maximum penalty parameter $\lambda$ defined in Equation 5 was set to 20 .

\footnotetext{
${ }^{2}$ http://www.bic.mni.mcgill.ca/brainweb/

${ }^{3}$ http://www.cma.mgh.harvard.edu/ibsr/
} 
Table 1. The mean values of $P$ and SDs of GM, WM and CSF with different methods. $B R$ denotes before registration, USSP (WOE) denotes using USSP features without the explicit anatomical energy term, USSP (WE) denotes using USSP features with the explicit anatomical energy term.

\begin{tabular}{|c|c|c|c|c|c|c|}
\hline Tissue & BR & FFD & Demons & HAMMER & USSP (WOE) & USSP (WE) \\
\hline Gray & $0.41923 \pm 0.07$ & $0.73816 \pm 0.06$ & $0.77416 \pm 0.05$ & $0.79563 \pm 0.06$ & $0.84281 \pm 0.05$ & $0.86725 \pm 0.04$ \\
White & $0.48344 \pm 0.03$ & $0.77825 \pm 0.04$ & $0.78024 \pm 0.06$ & $0.81093 \pm 0.02$ & $0.83527 \pm 0.08$ & $0.86103 \pm 0.07$ \\
CSF & $0.37025 \pm 0.06$ & $0.73072 \pm 0.06$ & $0.74281 \pm 0.03$ & $0.75682 \pm 0.02$ & $0.79093 \pm 0.06$ & $0.84774 \pm 0.03$ \\
\hline
\end{tabular}

\subsection{Experiments with Simulated Data}

In this section, the proposed method is evaluated on the simulated 3D T1 image data obtained from BrainWeb. 20 image volumes from different subjects were used. One of the image volumes was served as the template image, and the others were used as the subject images. Each image has resolution of $256 \times 256 \times 181$ voxels. The segmentation results are provided by BrainWeb. Before registration, the skull stripping process was performed on each image as it is a required step for HAMMER [1] to be compared in this paper. The software Brain Suite version 2 obtained from USC4 was used to accomplish the skull removing process.

Figures 4(b) to (e) show the reconstructed average brain images after the registration using FFD [3], Demons [4, HAMMER [1] and the proposed method (without explicit anatomical energy term). Figure 4 (a) is the template image for reference. The control point spacing of FFD is $2.5 \mathrm{~mm}$, as suggested in [16]. It is visually observed that the proposed method achieves the best registration accuracy as the average brain images obtained via the proposed method preserve most of the details of the template image and are sharper than those obtained by the other compared methods. The tissue overlap of gray matter (GM), white matter (WM) and cerebrospinal fluid (CSF) between the template and the transformed subject images is also adopted as the evaluation measure [16] to analyze the registration accuracy. It is defined as $P=\frac{N(A \cap B)}{N(A \cup B)}$, where $A$ and $B$ denote the regions of a specific tissue in two images. The average values of $P$ and the standard deviations of GM, WM and CSF before registration, registration after using FFD [3], Demons [4, HAMMER [1] and the proposed method with and without the explicit anatomical energy term are listed in Table 1. As observed in the Table 1, the proposed method achieves the highest value of $P$ among all the compared methods for the simulated 3D data sets. If the explicit anatomical energy term is used, the registration accuracy can be further improved.

\subsection{Experiments with Real Data}

In this section, the proposed method is further evaluated by performing registration experiments on the 3D real datasets obtained from IBSR. 20 skull-stripped image volumes with segmentation results are obtained. Each of them has resolution around $256 \times 256 \times 64$ voxels. The experimental settings are similar to

\footnotetext{
${ }^{4}$ http://brainsuite.usc.edu/
} 

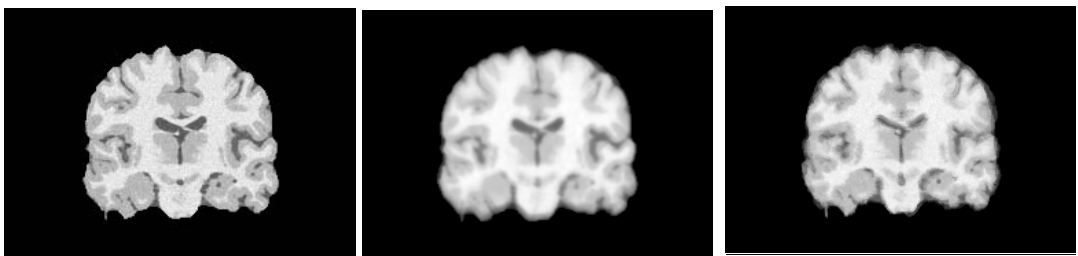

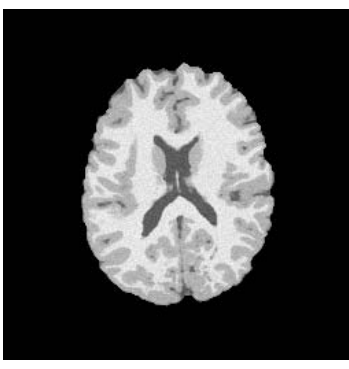

(a) Template

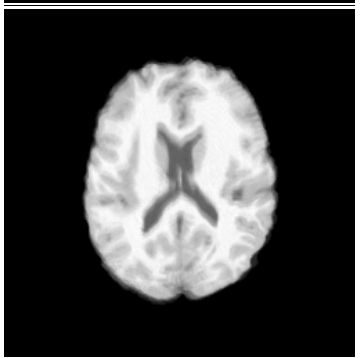

(b) FFD

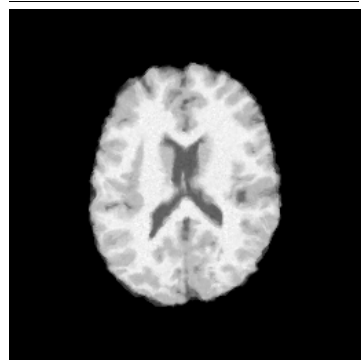

(c) Demons
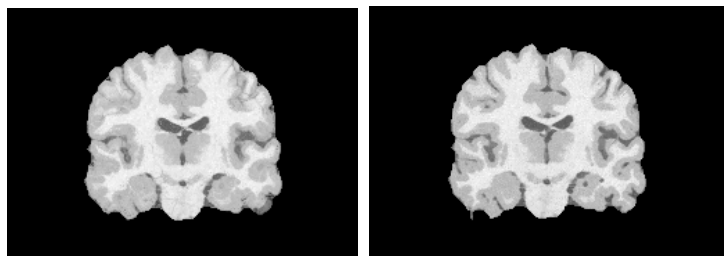

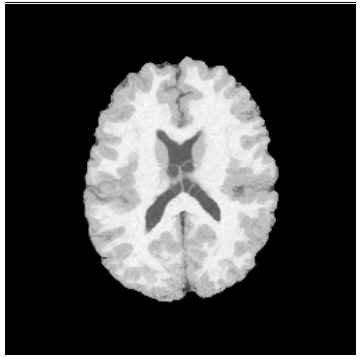

(d) Hammer

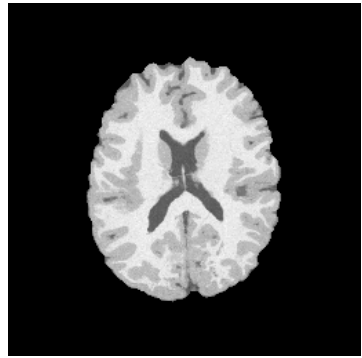

(e) Our Method

Fig. 4. (a) The template image; Average brain obtained using: (b) FFD, (c) Demons, (d) HAMMER and (e) the proposed method

the settings described in Section 4.1. The same cross section of the template image, the resulting average brain images obtained via various methods are shown in Figure 5. The control point spacing of FFD was again set to $2.5 \mathrm{~mm}$. It is visually observed from Figure [5 that the proposed method has the highest registration accuracy among all the compared methods, especially in the region of gyral crowns, sulcal roots and ventricles, which are important and salient regions of the brain anatomical structures. The tissue overlap measure values $P$ of different approaches are listed in Table 2. It is shown that USSP has the highest value of $P$, which echoes the visual results shown in Figure 5 , FFD [3], Demons 


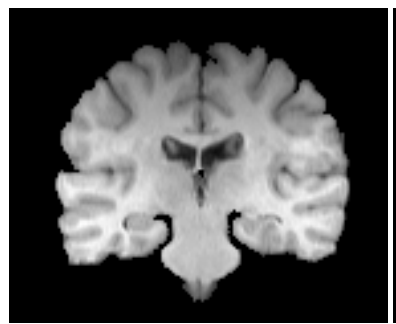

(a) Template

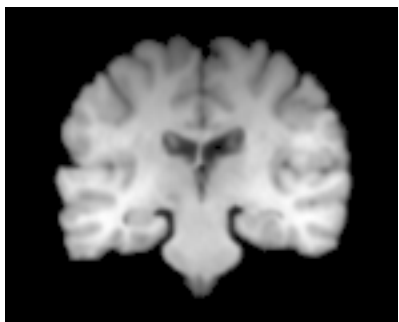

(b) FFD

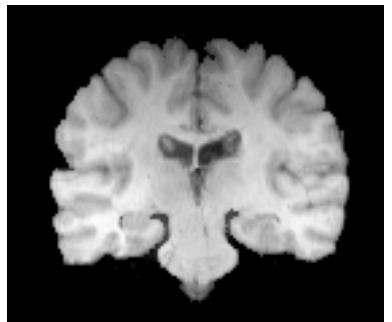

(c) Demons

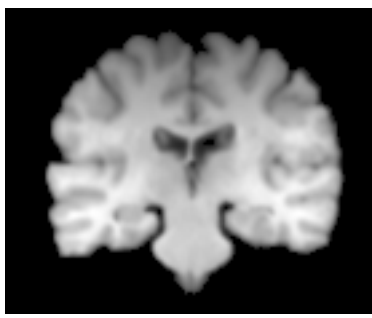

(d) Hammer

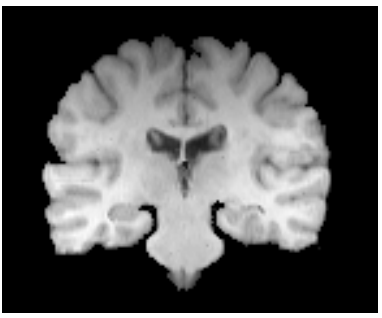

(e) Our Method

Fig. 5. (a) The template image; Average brain obtained using: (b) FFD, (c) Demons, (d) HAMMER and (e) the proposed method

Table 2. The mean values of $P$ and SDs of GM, WM and CSF with different methods. $B R$ denotes before registration, USSP (WOE) denotes using USSP features without the explicit anatomical energy term, USSP (WE) denotes using USSP features with the explicit anatomical energy term.

\begin{tabular}{|c|c|c|c|c|c|c|}
\hline Tissue & BR & FFD & Demons & HAMMER & USSP (WOE) & USSP (WE) \\
\hline Gray & $0.54082 \pm 0.06$ & $0.74034 \pm 0.04$ & $0.76477 \pm 0.03$ & $0.78174 \pm 0.05$ & $0.82073 \pm 0.04$ & $0.84624 \pm 0.05$ \\
White & $0.52147 \pm 0.05$ & $0.76285 \pm 0.06$ & $0.77830 \pm 0.04$ & $0.80627 \pm 0.05$ & $0.83816 \pm 0.06$ & $0.86216 \pm 0.06$ \\
CSF & $0.33094 \pm 0.07$ & $0.72192 \pm 0.04$ & $0.76693 \pm 0.04$ & $0.75906 \pm 0.04$ & $0.79631 \pm 0.03$ & $0.83772 \pm 0.04$ \\
\hline
\end{tabular}

[4], HAMMER [11] and the proposed method took about 7, 5, 10 and 11 hours respectively to register one image pair on a $3.2 \mathrm{GHz}$ P4 CPU with 2GB RAM.

\section{Conclusion}

In this paper, a new feature extraction method for non-rigid image registration is proposed. The uniform spherical structure pattern (USSP) feature is designed to extract monotonic gray-level transformation invariant and rotation invariant anatomical features from the input image volumes. The proposed feature can be extracted efficiently as the extraction process only requires several voxel-wise comparison operations. The registration problem is formulated as a Markov random field (MRF) labeling and energy minimization problem based on the USSP 
features. If the segmentation results of the input images are available, an explicit anatomical energy term can also be established easily based on the Fisher's separation criteria (FSC) measure function. From the experimental results on both the simulated and real 3D datasets, it is demonstrated that the proposed method gives the highest registration accuracy among all the compared methods.

\section{References}

1. Rohr, K.: Image registration based on thin plate splines and local estimates of anisotropic landmark localization uncertainties. In: Wells, W.M., Colchester, A.C.F., Delp, S.L. (eds.) MICCAI 1998. LNCS, vol. 1496, pp. 1174-1183. Springer, Heidelberg (1998)

2. Thompson, P., Toga, A.: A surface-based technique for warping three-dimensional images of the brain. TMI 15, 402-417 (1996)

3. Rueckert, D., Sonoda, L., et al.: Nonrigid registration using free-form deformations: Application to breast MR images. TMI 18, 712-721 (1999)

4. Thirion, J.: Image matching as a diffusion process: an analogy with maxwell's demons. MedI. A 2, 243-260 (1998)

5. Tu, Z., Narr, K., et al.: Brain anatomical structure segmentation by hybrid discriminative/generative models. TMI 27, 495-508 (2007)

6. Dirk, L., et al.: Nonrigid image registration using conditional mutual information. In: Karssemeijer, N., Lelieveldt, B. (eds.) IPMI 2007. LNCS, vol. 4584, pp. 725-737. Springer, Heidelberg (2007)

7. Tang, W., Chung, A.: Non-rigid image registration using graph-cuts. In: Ayache, N., Ourselin, S., Maeder, A. (eds.) MICCAI 2007, Part I. LNCS, vol. 4791, pp. 916-924. Springer, Heidelberg (2007)

8. Glocker, B., et al.: Inter and intra-modal deformable registration: Continuous deformations meet efficient optimal linear programming. In: Karssemeijer, N., Lelieveldt, B. (eds.) IPMI 2007. LNCS, vol. 4584, pp. 408-420. Springer, Heidelberg (2007)

9. Yershova, A., LaValle, S.: Deterministic sampling methods for spheres and so(3). In: ICRA, pp. 3974-3980 (2004)

10. Ojala, T., Pietikainen, M., Maenpaa, T.: Multiresolution gray-scale and rotation invariant texture classification with local binary patterns. PAMI 24, 971-987 (2002)

11. Shen, D., Davatzikos, C.: HAMMER: Hierarchical attribute matching mechanism for elastic registration. TMI 21, 1421-1439 (2002)

12. Wu, G., Qi, F., Shen, D.: Learning-based deformable registration of $\mathrm{mr}$ brain images. TMI 25, 1145-1157 (2006)

13. Fisher, A.: The Mathematical Theory of Probabilities. Macmillan, Basingstoke (1923)

14. Kruizinga, P., Petkov, N.: Nonlinear operator for oriented texture. TIP 8, 13951407 (1999)

15. Yuri, B., Olga, V., Ramin, Z.: Fast approximate energy minimization via graph cuts. PAMI 23, 1222-1239 (2001)

16. Crum, W., Rueckert, D., et al.: A framework for detailed objective comparison of non-rigid registration algorithms in neuroimaging. In: Barillot, C., Haynor, D.R., Hellier, P. (eds.) MICCAI 2004. LNCS, vol. 3216, pp. 679-686. Springer, Heidelberg (2004) 